\title{
Research Paper Heme oxygenase-1 gene promoter polymorphisms are associated with coronary heart disease and restenosis after percutaneous coronary intervention: a meta-analysis
}

\author{
Ming-Ming Zhang ${ }^{1}$, Ying-Ying Zheng ${ }^{2}$, Ying $\mathrm{Gao}^{3}$, Jing-Zhan Zhang ${ }^{2}$, Fen Liu ${ }^{2}$, Yi- \\ Ning Yang ${ }^{2}$, Xiao-Mei $\mathrm{Li}^{2}$, Yi-Tong $\mathrm{Ma}^{2}$, Xiang $\mathrm{Xie}^{2}$ \\ ${ }^{1}$ Department of Cardiology, Luoyang Central Hospital Affiliated to Zhengzhou University, Luoyang 471000, China \\ ${ }^{2}$ Heart Center, First Affiliated Hospital of Xinjiang Medical University, Urumqi, Xinjiang 830011, China \\ ${ }^{3}$ Department of Cadre ward, First Affiliated Hospital of Xinjiang Medical University, Urumqi, Xinjiang 830011, China \\ Correspondence to: Xiang Xie, email: xiangxie999@sina.com \\ Keywords: coronary heart disease, restenosis, heme oxygenase-1 gene, polymorphism, meta-analysis \\ Received: July 07, $2016 \quad$ Accepted: October 19, $2016 \quad$ Published: November 04, 2016
}

\section{ABSTRACT}

Numerous published studies have suggested that there is association between heme oxygenase-1 (HO-1) gene polymorphisms and coronary heart disease (CHD) or restenosis (RS) after percutaneous coronary intervention (PCI). This study aimed to clarify this association using a meta-analysis method. We used a systematic search for studies on the association of HO-1gene polymorphisms with CHD or RS in PubMed, Web of Science, the Cochrane Library, Wanfang Data and CNKI (China National Knowledge Infrastructure). We used Stata 12.0 software to perform the meta-analyses. Twenty-three studies, involving 12,130 patients with CHD or RS and 14,181 controls, were included. A statistically significant association between the HO1 (GT)n repeat length polymorphism and CHD was observed under allelic (odds ratio $(\mathrm{OR})=0.929,95 \%$ confidence interval $(\mathrm{CI})=0.881-0.978, p=0.005)$, recessive (OR $=0.858,95 \% \mathrm{CI}=0.780-0.945, p=0.002)$, and co-dominant $(\mathrm{OR}=0.843,95 \% \mathrm{CI}=$ $0.754-0.942, p=0.003$ ) models. Moreover, we also found a statistically significant association between the HO-1(GT)n repeat length polymorphism and RS under allelic (OR $=0.718,95 \% \mathrm{CI}=0.541-0.953, p=0.022)$ and co-dominant $(O R=0.522$, $95 \% \mathrm{CI}=0.306-0.889, p=0.017)$ models. We found a significant association of the HO-1T(-413)A single-nucleotide polymorphism (SNP) with CHD under allelic (OR = $0.915,95 \% C I=0.842-0.995, p=0.038)$, recessive $(\mathrm{OR}=0.869,95 \% \mathrm{CI}=0.760-$ $0.994, p=0.041)$, and co-dominant $(\mathrm{OR}=0.792,95 \% \mathrm{CI}=0.663-0.946, p=0.010)$ models. Our study indicates that both the HO-1(GT)n repeat length polymorphism and the T(-413)A SNP are associated with decreased risk of CHD. The (GT)n repeat length polymorphism was associated with RS following PCI.

\section{INTRODUCTION}

Coronary heart disease (CHD) is a multifactorial disorder resulting from the interaction between environmental and genetic factors [1]. Many genes that associate with CHD have been identified in recent years. In the treatment of $\mathrm{CHD}$, percutaneous coronary intervention (PCI) is the main therapy. However, restenosis (RS) following coronary stenting is a disadvantage of this therapy [2]. Current studies suggest that there are associations between genetic factors and the development of CHD or RS after PCI $[3,4]$.
Current studies have documented the interaction between HO-1 gene polymorphisms and CHD or RS after PCI. HO-1 is a subtype of heme oxygenase (HO) which plays a key regulatory role in the synthesis and catabolism of bilirubin [5]. HO will be increased significantly when the body responds to oxidative stress. During the degradation of heme to biliverdin, HO plays an important role as a rate-limiting enzyme [6]. Recently, two loci of HO-1 gene have been identified to be associated with CHD or RS in different population $[7,8]$. One is the (GT) $\mathrm{n}$ dinucleotide repeat length polymorphism, the other is the T(-413)A (rs2071746). Both loci are located in the 
HO-1 gene promoter region and influence serum HO-1 expression levels [8].

Although many studies on the relationship between these two loci and CHD have been carried out [9-20], the results are not conclusive. Some studies [9-16] have indicated that there is a positive correlation between the HO-1 (GT)n repeat length polymorphism and CHD, while other studies [17-20] have suggested that alterations in HO-1 expression play no obvious role in the pathogenesis of CHD. Several studies [22-23] have indicated that HO-1 genetic polymorphisms are associated with RS after PCI. However, the results of the subsequent studies [17, 24-26] do not support this result.

Based on these observations, two meta-analyses [27-28] related to this topic have been published. Qiao et al. [27] reported a positive correlation between genetic polymorphisms of HO-1 gene and CHD or RS after PCI. However, the meta-analysis from Rong et al. [28] do not support this results. Thus, the association of HO-1 gene polymorphisms with $\mathrm{CHD}$ or RS remains unclear. To clarify these associations, we performed an updated metaanalysis.

\section{RESULTS}

\section{Study characteristics}

There were 176 potentially relevant papers acquired from PubMed, Web of Science, the Cochrane Library, Wanfang and CNKI databases. Of these, we excluded 143 documents because of irrelevance to the aim of our study after reading the title and abstract. The remaining 33 documents were full-text reviewed, and 3 studies were excluded due to reported associations with diabetes [2931]. Four studies were not case-control studies [32-35], 3 studies were excluded because the variable number tandem repeat was different [36-38], 2 studies were excluded because there was no genotype data or it was a review [39-40]. Furthermore, 2 papers were excluded because of their lack of relation to CHD risk but rather to cardiovascular disease prognosis [41-42]. Finally, our meta-analysis included 19 eligible studies [9-26]. Table 1 and Table 2 listed the characteristics of each study. Finally, a total of 13 studies of the (GT)n repeat length polymorphism and 4 studies of the T(-413)A SNP with CHD were included. Six studies of the (GT)n repeat length polymorphism with RS were included. Because 4 papers included 2 studies, there were 23 studies included in final analysis.

\section{Meta-analysis}

\section{HO-1(GT)n repeat length polymorphism and CHD}

First, we investigated the relation between $\mathrm{HO}-$ 1(GT)n repeat length polymorphism and CHD. No significant heterogeneity was identified by H-test and
$\mathrm{I}^{2}$ test in any of the genetic models (Table 3 ), therefore, the fixed-effect model was used. Significant statistical association was found between HO-1(GT)n repeat length polymorphism and CHD risk under an allelic contrast (S vs. $\mathrm{L}, \mathrm{OR}=0.929,95 \% \mathrm{CI}=0.881-0.978, \mathrm{P}=0.005)$, the recessive genetic model ( $\mathrm{SS}$ vs. $\mathrm{SL}+\mathrm{LL}, \mathrm{OR}=0.858,95 \%$ $\mathrm{CI}=0.780-0.945, \mathrm{P}=0.002)$, and the co-dominant genetic model (SS vs. LL, OR $=0.843,95 \% \mathrm{CI}=0.754-0.942, \mathrm{P}=$ 0.003). Comparing to SL+LL and LL genotypes carriers, the CHD risk was significantly decreased among the SS genotype patients (Figure 1-5). However, we did not find significant association in the dominant genetic model (Table 4).

The second subgroup analysis was conducted according to ethnicity. The fixed-effects model was utilized to perform meta-analysis in all of the genetic models. We found patients with SS genotype have decreased CHD risk compared to SL+LL and LL genotype carriers in the Asian subgroup ( $\mathrm{S}$ vs. L, OR $=0.891,95 \% \mathrm{CI}=0.837-0.949$, $\mathrm{P}=0.000$; SS vs. $\mathrm{SL}+\mathrm{LL}, \mathrm{OR}=0.815,95 \% \mathrm{CI}=0.731-$ $0.909, \mathrm{P}=0.000 ; \mathrm{SS}+\mathrm{SL}$ vs. $\mathrm{LL}, \mathrm{OR}=0.887,95 \% \mathrm{CI}=$ $0.803-0.980, \mathrm{P}=0.018$; $\mathrm{SS}$ vs. $\mathrm{LL}, \mathrm{OR}=0.781,95 \% \mathrm{CI}=$ $0.686-0.890, \mathrm{P}=0.000)$. However, this association was not observed in Caucasian populations (Table 4).

In addition, we conducted subgroup analysis according to quality assessment. The fixed-effects model was used in all of the genetic models. Significantly decreased risk of CHD was found among individuals with the SS genotype compared to patients with L allele (SL + LL and LL genotypes) in the good-quality subgroup ( $\mathrm{S}$ vs. $\mathrm{L}, \mathrm{OR}=0.951,95 \% \mathrm{CI}=0.863-0.971, \mathrm{P}=0.003$; $\mathrm{SS}$ vs. $\mathrm{SL}+\mathrm{LL}, \mathrm{OR}=0.830,95 \% \mathrm{CI}=0.746-0.924, \mathrm{P}=$ 0.001 ; SS vs. LL, OR $=0.822,95 \% \mathrm{CI}=0.726-0.930, \mathrm{P}$ $=0.002$ ). However, this association was not found in the poor-quality reports (Table 4).

\section{HO-1(GT)n repeat length polymorphism and RS}

In 6 independent studies, drug-eluting stents were utilized. These studies examined the main baseline characteristics and identified no significant difference. First, significant heterogeneity was found in the contrast models, and therefore, the random-effects model was used in this meta-analysis. In the overall population, we found that patients with $\mathrm{S}$ allele had a decreased RS risk after PCI compared with the L allele carriers (S vs. L, OR $=0.718,95 \% \mathrm{CI}=0.541-0.953, \mathrm{P}=0.022 ; \mathrm{SS}$ vs. LL, $\mathrm{OR}=0.522,95 \% \mathrm{CI}=0.306-0.889, \mathrm{P}=0.017$ ) (Figure 6-7). However, we did not found significantly decreased risks of $\mathrm{RS}$ in other genetic models (SS vs. SL+LL, OR $=0.674,95 \% \mathrm{CI}=0.425-1.069, \mathrm{P}=0.093 ; \mathrm{SS}+\mathrm{SL}$ vs. $\mathrm{LL}$, $\mathrm{OR}=0.662,95 \% \mathrm{CI}=0.434-1.010, \mathrm{P}=0.056 ; \mathrm{SL}$ vs. $\mathrm{LL}$, $\mathrm{OR}=0.877,95 \% \mathrm{CI}=0.740-1.039, \mathrm{P}=0.130)$. Second, subgroup analysis was conducted according to ethnicity. The RS risk was significantly decreased among patients with the SS genotype compared with other genotypes in the Asian subgroup ( $\mathrm{S}$ vs. $\mathrm{L}, \mathrm{OR}=0.590,95 \% \mathrm{CI}=0.430$ - 
Table 1: Characteristics of included studies

\begin{tabular}{|c|c|c|c|c|c|c|c|c|c|c|c|c|c|c|c|c|c|}
\hline Reference & Year & Population & & Case & & & Contr & & & ge (years) & & $\begin{array}{l}\text { Genotyping } \\
\text { method }\end{array}$ & $\begin{array}{l}\text { Selection } \\
\text { criteria }\end{array}$ & HWE & $\begin{array}{l}\text { VNTR } \\
\text { Cut- } \\
\text { Off(s) } \\
(\geq)\end{array}$ & $\begin{array}{l}\text { NOS } \\
(\hbar)\end{array}$ & $\begin{array}{r}\text { Study } \\
\text { design }\end{array}$ \\
\hline $\begin{array}{l}\text { (GT)n } \\
\text { polymorphism } \\
\text { with CHD }\end{array}$ & & & Total & Male & female & Total & Male & female & Case & Control & $P$ & & & & & & \\
\hline Chen et al. & 2014 & East Asian & 386 & 358 & 28 & 361 & 300 & 61 & $70 \pm 8$ & $68 \pm 8$ & $>0.05$ & PCR-RFLP & CHD & 0.15 & 27 & 6 & $\mathrm{CC}$ \\
\hline Chen et al. & 2012 & East Asian & 2298 & 1675 & 623 & 2298 & 1675 & 623 & $\begin{array}{c}60.10 \pm \\
10.3\end{array}$ & $\begin{array}{c}59.9 \pm \\
10.2\end{array}$ & 0.62 & $\mathrm{CE}$ & CHD & 0.11 & 25 & 8 & $\mathrm{CC}$ \\
\hline Endler et al. & 2004 & Caucasian & 180 & 130 & 50 & 211 & 103 & 108 & $\begin{array}{c}57-72 \\
(64)\end{array}$ & $\begin{array}{c}48-67 \\
(58)\end{array}$ & 0.13 & $\begin{array}{c}\text { PCR- } \\
\mathrm{SSP}+\mathrm{CE}\end{array}$ & CHD & 0.91 & 25 & 6 & $\mathrm{CC}$ \\
\hline Funk et al. & 2004 & Caucasian & 399 & 187 & 212 & 398 & 192 & 206 & $\begin{array}{l}59-78 \\
(69)\end{array}$ & $\begin{array}{c}40-59 \\
(47)\end{array}$ & $<0.05$ & PCR-SSP & CHD & 0.90 & 25 & 6 & $\mathrm{CC}$ \\
\hline Gregorek et al. & 2013 & Caucasian & 59 & NA & NA & 58 & NA & NA & $\begin{array}{c}62-73 \\
(69)\end{array}$ & $\begin{array}{c}57-73 \\
(64)\end{array}$ & $>0.05$ & PCR-SSP & CHD & 0.85 & 25 & 6 & $\mathrm{CC}$ \\
\hline Han et al. & 2014 & East Asian & 110 & 71 & 39 & 107 & 56 & 51 & $\begin{array}{c}63 \pm \\
11\end{array}$ & $52 \pm 12$ & $<0.01$ & PCR-SSP & $\mathrm{CHD}$ & 0.06 & 25 & 6 & $\mathrm{CC}$ \\
\hline Kaneda et al. & 2002 & East Asian & 298 & 250 & 48 & 279 & 173 & 106 & $\begin{array}{c}63 \pm \\
0.5\end{array}$ & $58 \pm 0.7$ & $>0.05$ & PCR-SSP & CHD & 0.32 & 27 & 8 & $\mathrm{CC}$ \\
\hline $\begin{array}{l}\text { Lüblinghoff } \\
\text { et al. }\end{array}$ & 2009 & Caucasian & 2526 & 1891 & 635 & 693 & 360 & 333 & $\begin{array}{c}63 \pm \\
10\end{array}$ & $55 \pm 12$ & $>0.05$ & $\mathrm{CE}$ & CHD & 0.73 & 25 & 7 & $\mathrm{CC}$ \\
\hline $\begin{array}{l}\text { MI in Endler } \\
\text { et al. }\end{array}$ & 2004 & Caucasian & 258 & 199 & 59 & 211 & 103 & 108 & $\begin{array}{c}53- \\
71(60.5)\end{array}$ & $\begin{array}{c}48-67 \\
(58)\end{array}$ & 0.22 & $\begin{array}{c}\text { PCR- } \\
\text { SSP+CE }\end{array}$ & MI & 0.91 & 25 & 6 & $\mathrm{CC}$ \\
\hline $\begin{array}{l}\text { Schillinger } \\
\text { et al. }\end{array}$ & 2002 & Caucasian & 70 & 51 & 19 & 62 & 20 & 42 & $\begin{array}{c}62- \\
78\end{array}$ & $61-79$ & 0.40 & $\begin{array}{c}\text { PCR- } \\
\text { SSP+CE }\end{array}$ & CHD & 0.15 & 25 & 6 & $\mathrm{CC}$ \\
\hline Wang et al. & 2009 & $\begin{array}{l}\text { Middle } \\
\text { Asian }\end{array}$ & 287 & 177 & 110 & 190 & 126 & 64 & $\begin{array}{c}58.42 \pm \\
11.1\end{array}$ & $\begin{array}{c}58.03 \pm \\
10 . \\
4\end{array}$ & 0.34 & PCR-SSP & MI & 0.82 & 27 & 7 & $\mathrm{CC}$ \\
\hline $\begin{array}{l}\text { Y. H. Chen } \\
\text { et al. }\end{array}$ & 2008 & East Asian & 664 & 611 & 53 & 322 & 264 & 58 & $\begin{array}{c}69 \pm \\
9\end{array}$ & $67 \pm 7$ & $>0.05$ & $\mathrm{CE}$ & CHD & 0.49 & 27 & 8 & $\mathrm{CC}$ \\
\hline Zhang et al. & 2010 & East Asian & 300 & 228 & 72 & 182 & 106 & 76 & $\begin{array}{c}62.96 \pm \\
12.1\end{array}$ & $\begin{array}{c}64.23 \pm \\
12.1\end{array}$ & 0.13 & $\mathrm{CE}$ & CHD & 0.98 & 25 & 7 & $\mathrm{CC}$ \\
\hline \multicolumn{18}{|l|}{$\begin{array}{l}(G T) n \\
\text { polymorphism } \\
\text { with restenosis }\end{array}$} \\
\hline Exner et al & 2001 & Caucasian & 23 & NA & NA & 73 & NA & NA & $\begin{array}{c}60-72 \\
(70)\end{array}$ & $\begin{array}{c}63-72 \\
(69)\end{array}$ & 0.10 & PCR-SSP & CHD & 0.02 & 25 & 7 & $\mathrm{CS}$ \\
\hline Han et al. & 2014 & East Asian & 18 & NA & NA & 27 & NA & NA & $63 \pm 11$ & $52 \pm 12$ & $<0.01$ & PCR-RFLP & $\mathrm{CHD}$ & 0.07 & 25 & 6 & $\mathrm{CS}$ \\
\hline Klaus et al. & 2007 & Caucasian & 401 & NA & NA & 956 & NA & NA & $\begin{array}{c}65.5 \pm \\
10.8\end{array}$ & $\begin{array}{c}66.2 \pm \\
10.7\end{array}$ & 0.51 & PCR-SSP & CHD & 0.01 & 25 & 7 & $\mathrm{CS}$ \\
\hline $\begin{array}{l}\text { Schillinger } \\
\text { et al. }\end{array}$ & 2004 & Caucasian & 95 & NA & NA & 183 & NA & NA & $\begin{array}{c}61-78 \\
(71)\end{array}$ & $\begin{array}{c}66-78 \\
(73)\end{array}$ & 0.37 & PCR-SSP & CHD & 0.58 & 25 & 7 & $\mathrm{CS}$ \\
\hline Wijpkema et al. & 2006 & Caucasian & 324 & NA & NA & 2601 & NA & NA & NA & NA & NA & PCR-SSP & CHD & 0.17 & 25 & 6 & $\mathrm{CS}$ \\
\hline $\begin{array}{l}\text { Y. H. Chen } \\
\text { et al. }\end{array}$ & 2003 & East Asian & 111 & NA & NA & 212 & NA & NA & $70 \pm 8$ & $68 \pm 9$ & 0.07 & $\mathrm{CE}$ & CHD & 0.89 & 26 & 7 & $\mathrm{CS}$ \\
\hline \multicolumn{18}{|l|}{$\begin{array}{l}\text { T(-413)A } \\
\text { polymorphism } \\
\text { with CHD }\end{array}$} \\
\hline $\begin{array}{l}\text { Lüblinghoff } \\
\text { et al. }\end{array}$ & 2009 & Caucasian & 2526 & 1891 & 635 & 693 & 360 & 333 & $63 \pm 10$ & $55 \pm 12$ & $>0.05$ & PCR-RFLP & CHD & 0.49 & NA & 7 & $\mathrm{CC}$ \\
\hline MI in Ono et al. & 2004 & East Asian & 393 & 326 & 67 & 1972 & 946 & 1026 & $\begin{array}{l}58.4 \\
\pm 0.6\end{array}$ & $\begin{array}{c}59.9 \pm \\
0.3\end{array}$ & 0.06 & PCR-SSP & MI & 0.04 & NA & 8 & $\mathrm{CC}$ \\
\hline Ono et al. & 2004 & East Asian & 204 & 169 & 35 & 1972 & 946 & 1026 & $\begin{array}{c}59.7 \pm \\
0.8\end{array}$ & $\begin{array}{c}59.9 \pm \\
0.3\end{array}$ & 0.07 & PCR-SSP & $\mathrm{CHD}$ & 0.04 & NA & 8 & $\mathrm{CC}$ \\
\hline Zhang et al. & 2010 & East Asian & 200 & 168 & 32 & 120 & 100 & 20 & $\begin{array}{c}61.17 \pm \\
5.6\end{array}$ & $\begin{array}{c}62.68 \pm \\
6.1\end{array}$ & 0.23 & PCR-RFLP & $\mathrm{CHD}$ & 0.89 & NA & 7 & $\mathrm{CC}$ \\
\hline
\end{tabular}

Notes: CC, case-control study; CS, Cohort study; VNTR, variable number tandem repeat; HWE, Hardy-Weinberg equilibrium; CHD, coronary heart disease; MI, myocardial infarction; NOS, Newcastle-Ottawa Scale; PCR, polymerase chain reaction; RFLP, restriction fragment length polymorphism; SSP, sequence-specific primers; CE, capillary electrophoresis. 
Table 2: Date characteristics of included studies

\begin{tabular}{|c|c|c|c|c|c|c|c|c|c|c|c|c|c|c|}
\hline \multirow{3}{*}{ Reference } & \multirow{3}{*}{ Year } & \multirow{3}{*}{ Ethnicity } & \multicolumn{6}{|c|}{ Case } & \multicolumn{6}{|c|}{ Control } \\
\hline & & & \multirow[t]{2}{*}{$\mathbf{N}$} & \multicolumn{3}{|c|}{ Genotype (n) } & \multicolumn{2}{|c|}{ allele } & \multirow[t]{2}{*}{$\mathbf{N}$} & \multicolumn{3}{|c|}{ Genotype (n) } & \multicolumn{2}{|c|}{ allele } \\
\hline & & & & SS & SL & $\mathbf{L L}$ & $\mathbf{S}$ & $\mathbf{L}$ & & SS & SL & $\mathbf{L L}$ & $\mathbf{S}$ & $\mathbf{L}$ \\
\hline \multicolumn{15}{|l|}{$\begin{array}{l}\text { (GT)n } \\
\text { polymorphism } \\
\text { with CHD }\end{array}$} \\
\hline Chen et al. & 2014 & East Asian & 386 & 94 & 187 & 105 & 375 & 397 & 361 & 78 & 194 & 89 & 350 & 372 \\
\hline Chen et al. & 2012 & East Asian & 2298 & 436 & 1268 & 594 & 2140 & 2456 & 2298 & 548 & 1187 & 563 & 2283 & 2313 \\
\hline Endler et al. & 2004 & Caucasian & 180 & 12 & 74 & 94 & 98 & 262 & 211 & 16 & 83 & 112 & 115 & 307 \\
\hline Funk et al. & 2004 & Caucasian & 399 & 39 & 180 & 180 & 258 & 540 & 398 & 46 & 177 & 175 & 269 & 527 \\
\hline Gregorek et al. & 2013 & Caucasian & 59 & 7 & 35 & 17 & 49 & 69 & 58 & 10 & 29 & 19 & 49 & 67 \\
\hline Han et al. & 2014 & East Asian & 110 & 10 & 46 & 54 & 66 & 154 & 107 & 7 & 56 & 44 & 70 & 144 \\
\hline Kaneda et al. & 2002 & East Asian & 298 & 47 & 165 & 86 & 259 & 337 & 279 & 48 & 145 & 86 & 241 & 317 \\
\hline Lüblinghoff et al. & 2009 & Caucasian & 2526 & 286 & 1070 & 1170 & 1642 & 3410 & 693 & 66 & 302 & 325 & 434 & 952 \\
\hline MI in Endler et al. & 2004 & Caucasian & 258 & 13 & 106 & 139 & 132 & 384 & 211 & 16 & 83 & 112 & 115 & 307 \\
\hline Schillinger et al. & 2002 & Caucasian & 70 & 9 & 38 & 23 & 56 & 84 & 62 & 4 & 32 & 26 & 40 & 84 \\
\hline Wang et al. & 2009 & $\begin{array}{l}\text { Middle } \\
\text { Asian }\end{array}$ & 287 & 57 & 128 & 102 & 242 & 332 & 190 & 55 & 93 & 42 & 203 & 177 \\
\hline Y. H. Chen et al. & 2008 & East Asian & 664 & 147 & 322 & 195 & 616 & 712 & 322 & 74 & 167 & 81 & 315 & 329 \\
\hline \multicolumn{15}{|l|}{$\begin{array}{l}(\mathrm{GT}) \mathrm{n} \\
\text { polymorphism } \\
\text { with restenosis }\end{array}$} \\
\hline Exner et al & 2001 & Caucasian & 23 & 1 & 8 & 14 & 10 & 36 & 73 & 7 & 45 & 21 & 59 & 87 \\
\hline Han et al. & 2014 & East Asian & 18 & 1 & 5 & 12 & 7 & 29 & 27 & 0 & 14 & 13 & 14 & 40 \\
\hline Klaus et al. & 2007 & Caucasian & 401 & 45 & 155 & 201 & 245 & 557 & 956 & 109 & 370 & 477 & 588 & 1324 \\
\hline Schillinger et al. & 2004 & Caucasian & 95 & 3 & 33 & 59 & 39 & 151 & 183 & 20 & 86 & 77 & 126 & 240 \\
\hline Wijpkema et al. & 2006 & Caucasian & 324 & 151 & 151 & 22 & 453 & 195 & 2601 & 1256 & 1124 & 221 & 3636 & 1566 \\
\hline Y. H. Chen et al. & 2003 & East Asian & 111 & 11 & 60 & 40 & 82 & 140 & 212 & 54 & 105 & 53 & 213 & 211 \\
\hline $\begin{array}{l}\text { T(-413)A } \\
\text { polymorphism } \\
\text { with CHD }\end{array}$ & & & & $\mathrm{AA}$ & AT & $\mathrm{TT}$ & A & $\mathrm{T}$ & & AA & AT & $\mathrm{TT}$ & A & $\mathrm{T}$ \\
\hline Lüblinghoff et al. & 2009 & Caucasian & 2526 & 893 & 1181 & 452 & 2967 & 2085 & 693 & 246 & 341 & 106 & 833 & 553 \\
\hline MI in Ono et al. & 2004 & East Asian & 393 & 64 & 208 & 121 & 336 & 450 & 1972 & 420 & 930 & 622 & 1770 & 2174 \\
\hline Ono et al. & 2004 & East Asian & 204 & 32 & 101 & 71 & 165 & 243 & 1972 & 420 & 930 & 622 & 1770 & 2174 \\
\hline Zhang et al. & 2010 & East Asian & 200 & 40 & 137 & 23 & 217 & 183 & 120 & 28 & 80 & 12 & 136 & 104 \\
\hline
\end{tabular}

$0.809, \mathrm{P}=0.001 ; \mathrm{SS}$ vs. $\mathrm{SL}+\mathrm{LL}, \mathrm{OR}=0.755,95 \% \mathrm{CI}$ $=0.065-0.737, \mathrm{P}=0.022 ; \mathrm{SS}+\mathrm{SL}$ vs. $\mathrm{LL}, \mathrm{OR}=0.572$, $95 \% \mathrm{CI}=0.361-0.907, \mathrm{P}=0.018 ; \mathrm{SS}$ vs. $\mathrm{LL}, \mathrm{OR}=0.548$, $95 \% \mathrm{CI}=0.461-0.660, \mathrm{P}=0.003)$. When we excluded the studies which were inconsistent with the HWE, the protective effects of the $\mathrm{S}$ allele for RS after PCI persisted ( $\mathrm{S}$ vs. $\mathrm{L}, \mathrm{OR}=0.679,95 \% \mathrm{CI}=0.446-0.934, \mathrm{P}=0.041$; $\mathrm{SS}$ vs. $\mathrm{LL}, \mathrm{OR}=0.414,95 \% \mathrm{CI}=0.195-0.879, \mathrm{P}=0.022)$ (Table 4).

\section{HO-1 T(-413)A SNP and CHD risk}

There were 4 studies that investigated the relationship between the HO-1T(-413)A SNP and CHD. No significant heterogeneity was found in the contrast models, and so the fixed-effect model was used in this of meta-analysis. Meta-analysis suggested that there was a significant association between the HO-1 T(-413)A polymorphism and CHD under the allele contrast (A vs. $\mathrm{T}, \mathrm{OR}=0.915,95 \% \mathrm{CI}=0.842-0.995, \mathrm{P}=0.038)$, the 
Table 3: heterogeneity test analysis -(GT)n repeat length polymorphism with CHD

\begin{tabular}{lccc}
\hline \multirow{2}{*}{ Study } & \multicolumn{3}{c}{ Heterogeneity test } \\
\cline { 2 - 3 } & $\boldsymbol{H}$ & $\boldsymbol{I}^{\mathbf{2}}$ & $\boldsymbol{P}$ \\
\hline Allele model(S/L) & 1.21 & $31.40 \%$ & 0.132 \\
Recessive model(SS/SL+LL) & 1.27 & $38.30 \%$ & 0.078 \\
Dominant model(SS+SL/LL) & 1.07 & $12.40 \%$ & 0.320 \\
Co-dominant model(SL/LL) & 1.01 & $2.70 \%$ & 0.420 \\
Co-dominant model(SS/LL) & 1.25 & $35.90 \%$ & 0.096 \\
\hline
\end{tabular}

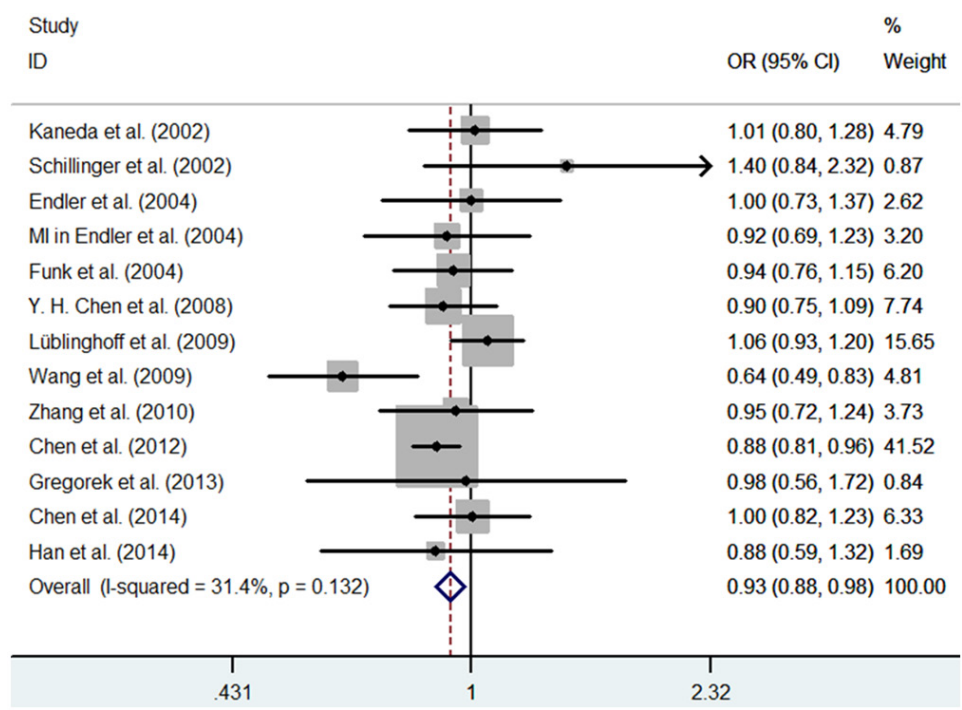

Figure 1: Meta-analysis of the relationship between the (GT)n polymorphism in the HO-1 gene and CHD risk for the allele model $(\mathrm{S} / \mathrm{L})$.

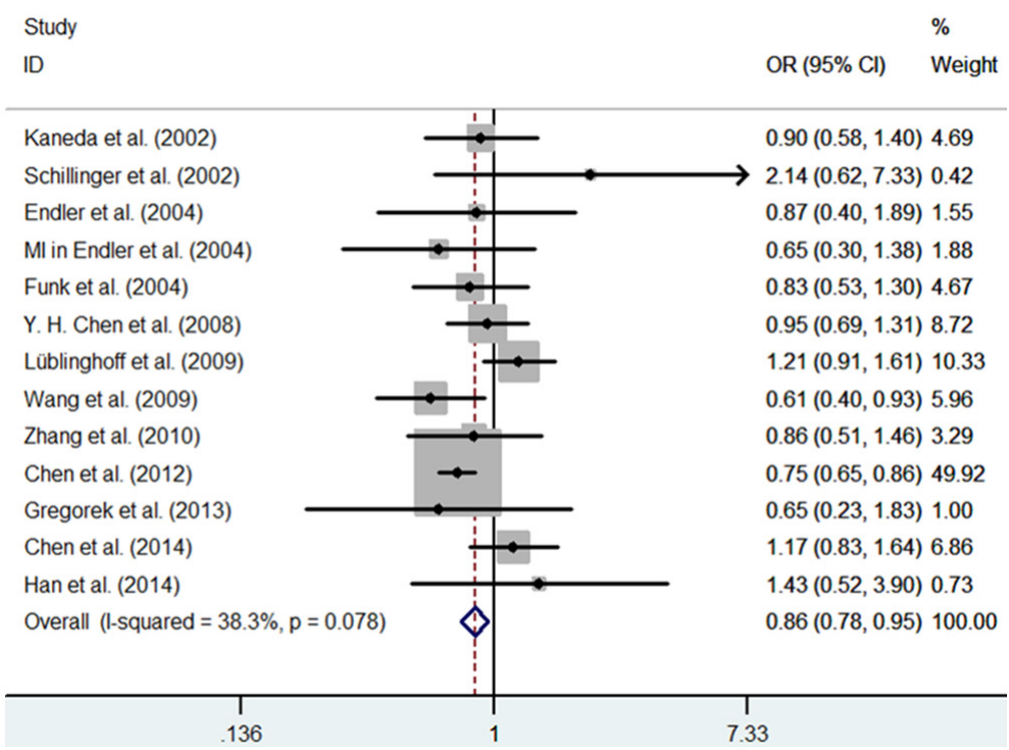

Figure 2: Meta-analysis of the relationship between the (GT)n polymorphism in the HO-1 gene and CHD risk for the recessive model (SS/SL+LL). 


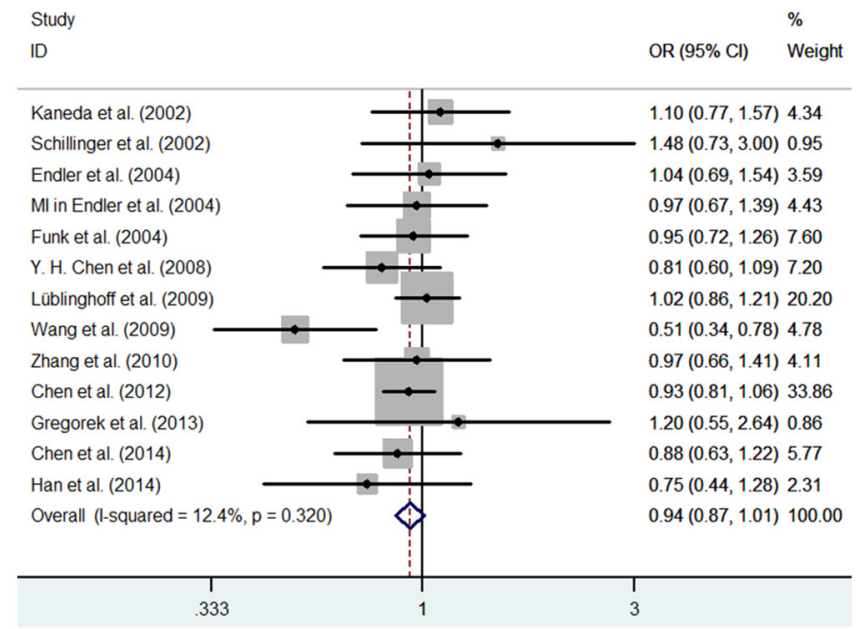

Figure 3: Meta-analysis of the relationship between the (GT)n polymorphism in the HO-1 gene and CHD risk for the dominant model (SS+SL/LL).

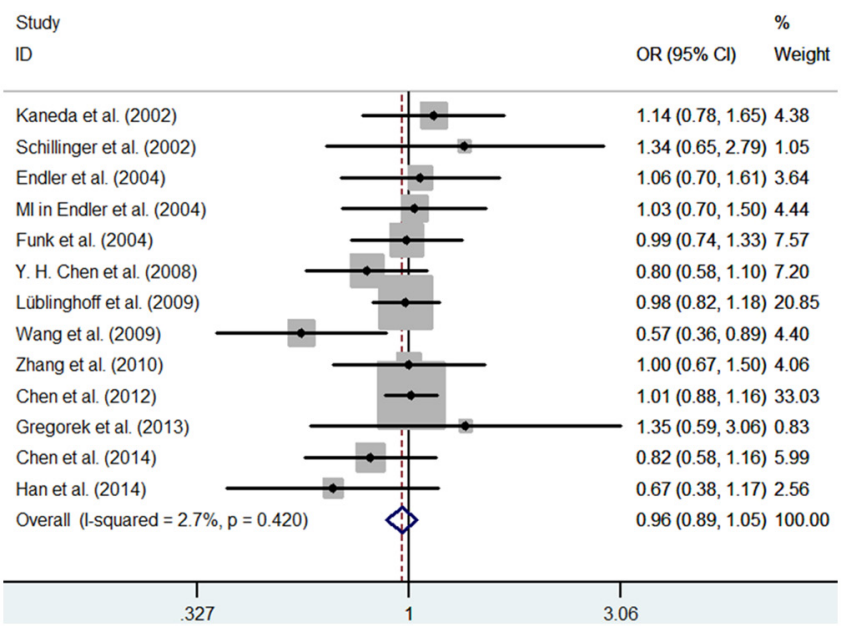

Figure 4: Meta-analysis of the relationship between the (GT)n polymorphism in the HO-1 gene and CHD risk for the co-dominant model (SL/LL).

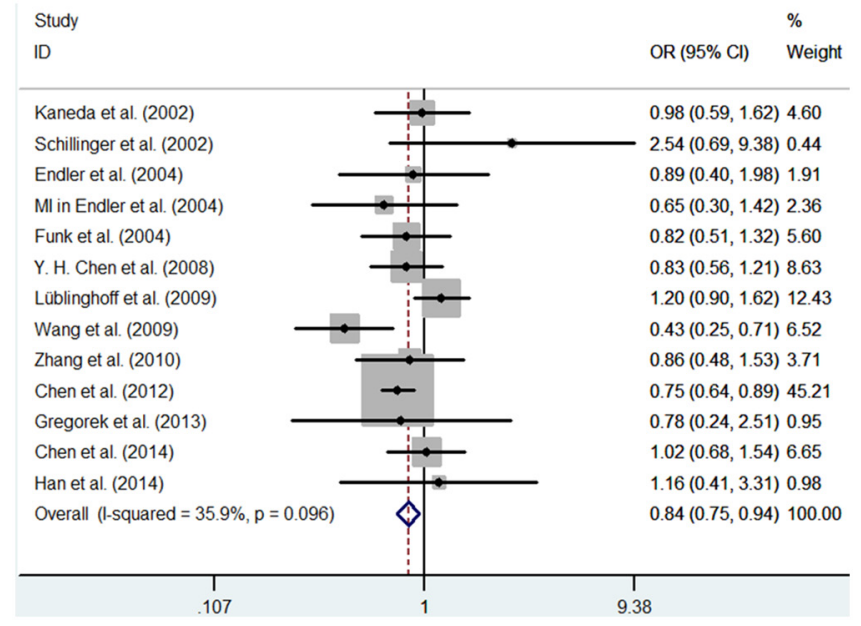

Figure 5: Meta-analysis of the relationship between the (GT)n polymorphism in the HO-1 gene and CHD risk for the co-dominant model (SS/LL). 
Table 4: Results From a Meta-Analysis of the Association Between coronary heart disease or restenosis after PCI and the Heme oxygenase-1 gene promoter polymorphism

\begin{tabular}{|c|c|c|c|c|c|c|c|c|c|c|c|c|c|}
\hline \multirow{3}{*}{$\begin{array}{l}\text { Polymorphism } \\
\text { and Subgroup }\end{array}$} & \multirow{3}{*}{$\begin{array}{c}\text { No. of } \\
\text { Studies }\end{array}$} & \multirow{3}{*}{$\begin{array}{l}\text { No. of } \\
\text { Cases }\end{array}$} & \multirow{3}{*}{$\begin{array}{l}\text { No. of } \\
\text { Controls }\end{array}$} & \multicolumn{10}{|c|}{ Genotype } \\
\hline & & & & \multicolumn{2}{|l|}{$\mathbf{S} / \mathbf{L}$} & \multicolumn{2}{|c|}{ SS/SL+LL } & \multicolumn{2}{|c|}{$\mathbf{S S}+\mathbf{S L} / \mathbf{L L}$} & \multicolumn{2}{|l|}{ SL/LL } & \multicolumn{2}{|c|}{ SS/LL } \\
\hline & & & & $\begin{array}{l}\text { OR and } \\
95 \% C I\end{array}$ & $P$ Value & $\begin{array}{l}\text { OR and } \\
95 \% C I\end{array}$ & $P$ Value & $\begin{array}{l}\text { OR and } \\
95 \% C I\end{array}$ & $P$ Value & $\begin{array}{l}\text { OR and } \\
95 \% C I\end{array}$ & $P$ Value & $\begin{array}{l}\text { OR and } \\
95 \% C I\end{array}$ & $P$ Value \\
\hline \multicolumn{14}{|l|}{$\begin{array}{l}\text { (GT)n } \\
\text { polymorphism } \\
\text { with CHD }\end{array}$} \\
\hline Total & 13 & 7835 & 5372 & $\begin{array}{c}0.929(0.881 \\
0.978)\end{array}$ & 0.005 & $\begin{array}{c}0.858(0.780 \\
0.945)\end{array}$ & 0.002 & $\begin{array}{c}0.937(0.867 \\
1.012)\end{array}$ & 0.100 & $\begin{array}{c}0.963(0.888 \\
1.045)\end{array}$ & 0.369 & $\begin{array}{c}0.843(0.754 \\
0.942)\end{array}$ & 0.003 \\
\hline Caucasian & 6 & 3492 & 1633 & $\begin{array}{c}1.019 \\
(0.927 \\
1.119)\end{array}$ & 0.701 & $\begin{array}{c}1.033(0.840 \\
1.271)\end{array}$ & 0.759 & $\begin{array}{c}1.020(0.901 \\
1.154)\end{array}$ & 0.756 & $\begin{array}{c}1.015(0.892 \\
1.156)\end{array}$ & 0.818 & $\begin{array}{c}1.042(0.838 \\
1.296)\end{array}$ & 0.709 \\
\hline Asian & 7 & 4343 & 3739 & $\begin{array}{c}0.891 \\
(0.837 \\
0.949)\end{array}$ & 0.000 & $\begin{array}{c}0.815(0.731 \\
0.909)\end{array}$ & 0.000 & $\begin{array}{c}0.887(0.803 \\
0.980)\end{array}$ & 0.018 & $\begin{array}{c}0.931(0.838 \\
1.034)\end{array}$ & 0.180 & $\begin{array}{c}0.781(0.686 \\
0.890)\end{array}$ & 0.000 \\
\hline Good quality & 6 & 6373 & 3964 & $\begin{array}{c}0.915 \\
(0.863 \\
0.971)\end{array}$ & 0.003 & $\begin{array}{c}0.830(0.746 \\
0.924)\end{array}$ & 0.001 & $\begin{array}{c}0.929(0.849 \\
1.017)\end{array}$ & 0.110 & $\begin{array}{c}0.964(0.877 \\
1.060)\end{array}$ & 0.551 & $\begin{array}{c}0.822(0.726 \\
0.930)\end{array}$ & 0.002 \\
\hline Poor quality & 7 & 1462 & 1408 & $\begin{array}{c}0.959 \\
(0.856 \\
1.074)\end{array}$ & 0.468 & $\begin{array}{c}0.995(0.795 \\
1.246)\end{array}$ & 0.976 & $\begin{array}{c}0.960(0.824 \\
1.117)\end{array}$ & 0.595 & $\begin{array}{c}0.961(0.819 \\
1.127)\end{array}$ & 0.623 & $\begin{array}{c}0.935(0.728 \\
1.201)\end{array}$ & 0.599 \\
\hline \multicolumn{14}{|l|}{$\begin{array}{l}(\mathrm{GT}) \mathrm{n} \\
\text { polymorphism } \\
\text { with restenosis }\end{array}$} \\
\hline Total & 6 & 972 & 4052 & $\begin{array}{c}0.718 \\
(0.541 \\
0.953)\end{array}$ & 0.022 & $\begin{array}{c}0.674(0.425 \\
1.069)\end{array}$ & 0.093 & $\begin{array}{c}0.662(0.434 \\
1.010)\end{array}$ & 0.056 & $\begin{array}{c}0.877(0.740 \\
1.039)\end{array}$ & 0.130 & $\begin{array}{c}0.522(0.306 \\
0.889)\end{array}$ & 0.017 \\
\hline Caucasian & 4 & 843 & 3813 & $\begin{array}{c}0.766 \\
(0.557 \\
1.053)\end{array}$ & 0.100 & $\begin{array}{c}0.870(0.637 \\
1.190)\end{array}$ & 0.384 & $\begin{array}{c}0.694(0.400 \\
1.204)\end{array}$ & 0.194 & $\begin{array}{c}0.742(0.439 \\
1.254)\end{array}$ & 0.265 & $\begin{array}{c}0.72(0.384 \\
1.380)\end{array}$ & 0.330 \\
\hline Asian & 2 & 129 & 239 & $\begin{array}{c}0.590 \\
(0.430 \\
0.809)\end{array}$ & 0.001 & $\begin{array}{c}0.755(0.065 \\
0.737)\end{array}$ & 0.022 & $\begin{array}{c}0.572(0.361 \\
0.907)\end{array}$ & 0.018 & $\begin{array}{c}0.689(0.426 \\
1.115)\end{array}$ & 0.130 & $\begin{array}{c}0.548(0.461 \\
0.660)\end{array}$ & 0.003 \\
\hline $\begin{array}{l}\text { Meeting } \\
\text { HWE }\end{array}$ & 4 & 548 & 3023 & $\begin{array}{c}0.679(0.446 \\
0.934)\end{array}$ & 0.041 & $\begin{array}{c}0.553(0.230 \\
1.327)\end{array}$ & 0.184 & $\begin{array}{c}0.664(0.381 \\
1.156)\end{array}$ & 0.148 & $\begin{array}{c}0.740(0.435 \\
1.258)\end{array}$ & 0.266 & $\begin{array}{c}0.414(0.195 \\
0.879)\end{array}$ & 0.022 \\
\hline $\begin{array}{l}\text { Deviating } \\
\text { from HWE }\end{array}$ & 2 & 424 & 1029 & $\begin{array}{c}0.693(0.296 \\
1.620)\end{array}$ & 0.397 & $\begin{array}{c}0.959(0.667 \\
1.380)\end{array}$ & 0.822 & $\begin{array}{c}0.554(0.151 \\
2.034)\end{array}$ & 0.373 & $\begin{array}{c}0.566(0.158 \\
2.209)\end{array}$ & 0.382 & $\begin{array}{c}0.684(0.192 \\
2.434)\end{array}$ & 0.557 \\
\hline $\begin{array}{l}\text { T(-413)A } \\
\text { polymorphism } \\
\text { with CHD }\end{array}$ & & & & \multicolumn{2}{|l|}{$\mathrm{A} / \mathrm{T}$} & \multicolumn{2}{|c|}{$\mathrm{AA} / \mathrm{AT}+\mathrm{TT}$} & \multicolumn{2}{|c|}{$\mathrm{AA}+\mathrm{AT} / \mathrm{TT}$} & \multicolumn{2}{|l|}{$\mathrm{AT} / \mathrm{TT}$} & \multicolumn{2}{|c|}{$\mathrm{AA} / \mathrm{TT}$} \\
\hline \multirow[t]{2}{*}{ Total } & & & & $\begin{array}{l}\text { OR and } \\
95 \% \mathrm{CI}\end{array}$ & $P$ Value & $\begin{array}{l}\text { OR and } \\
95 \% \mathrm{CI}\end{array}$ & $P$ Value & $\begin{array}{l}\text { OR and } \\
95 \% \mathrm{CI}\end{array}$ & $P$ Value & $\begin{array}{l}\text { OR and } \\
95 \% \mathrm{CI}\end{array}$ & $P$ Value & $\begin{array}{l}\text { OR and } \\
95 \% \mathrm{CI}\end{array}$ & $P$ Value \\
\hline & 4 & 3323 & 4757 & $\begin{array}{c}0.915(0.842 \\
0.995)\end{array}$ & 0.038 & $\begin{array}{c}0.869(0.760 \\
0.994)\end{array}$ & 0.041 & $\begin{array}{c}0.907(0.788 \\
1.045)\end{array}$ & 0.177 & $\begin{array}{c}0.958(0.826 \\
1.110)\end{array}$ & 0.567 & $\begin{array}{c}0.792(0.663 \\
0.946)\end{array}$ & 0.010 \\
\hline
\end{tabular}

recessive genetic model (AA vs. AT $+\mathrm{TT}, \mathrm{OR}=0.869,95 \%$ $\mathrm{CI}=0.760-0.994, \mathrm{P}=0.041)$, and the co-dominant genetic model (AA vs. TT, OR $=0.792,95 \% \mathrm{CI}=0.663-0.946, \mathrm{P}$ $=0.010)$ (Table 4).

\section{Sensitivity analysis}

We performed the sensitivity analysis to examine the influence of each study on the pooled ORs by deleting each study one at a time in each genetic model. The pooled
ORs showed no significant change, suggesting the results are stable (Figure 8).

\section{Publication bias}

In the present study, we utilized Egger's test and funnel plots to evaluate the publication bias of all contrast models. By Egger's test and funnel plots, we did not found publication biases for both the (GT)n repeat length polymorphism and T(-413)A SNP (Table 5, Figure 9). 


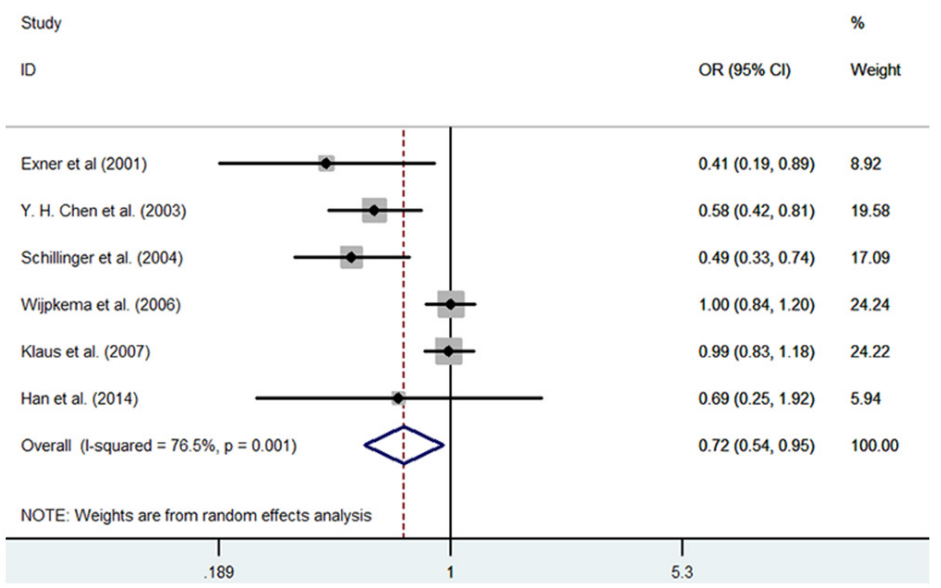

Figure 6: Meta-analysis of the relationship between the (GT)n polymorphism in the HO-1 gene and RS after PCI for the allele model $(\mathrm{S} / \mathrm{L})$.

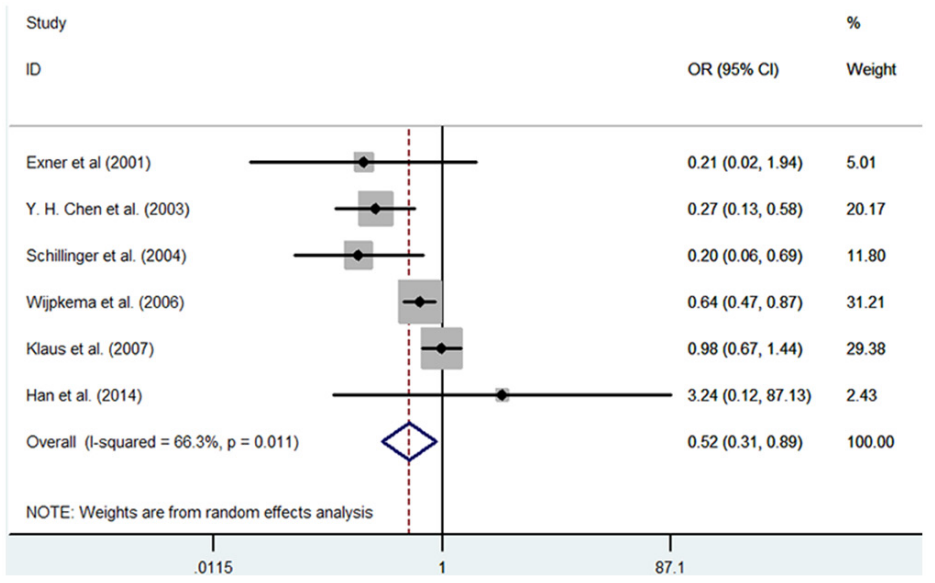

Figure 7: Meta-analysis of the relationship between the (GT)n polymorphism in the HO-1 gene and RS after PCI for the allele model (SS/LL).

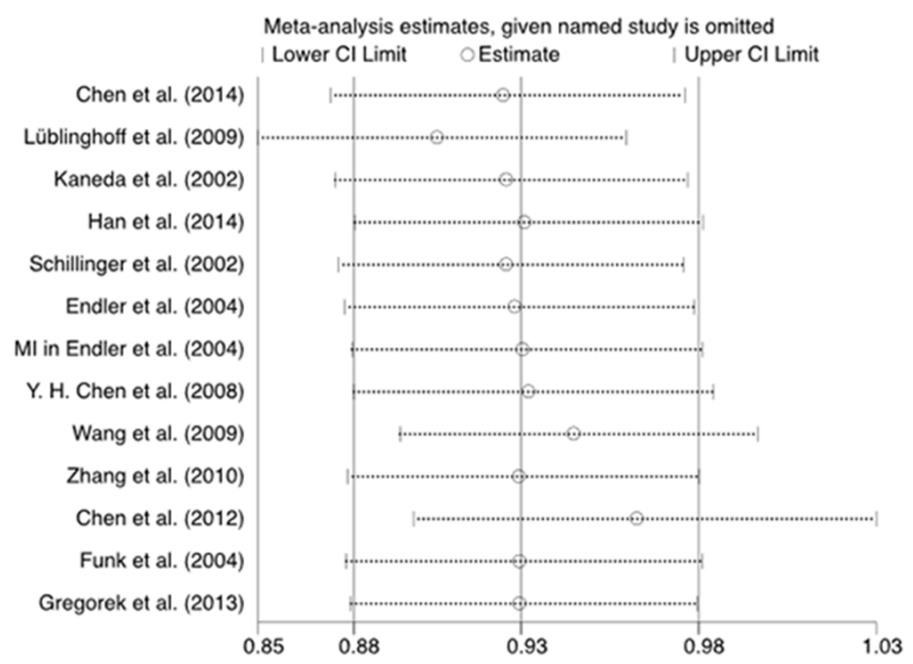

Figure 8: Sensitivity analysis of the relationship between the (GT)n polymorphism in the HO-1 gene and CHD risk for the allele model. 
Table 5: Egg's test results

\begin{tabular}{lcc}
\hline Association & Genetic model & $\boldsymbol{P}$ value \\
\hline (GT)n polymorphism and CHD & S versus L & 0.598 \\
& SS versus SL+LL & 0.301 \\
& SS+SL versus LL & 0.823 \\
(GT)n polymorphism and RS after PCI & SL versus LL & 0.975 \\
& SS versus LL & 0.519 \\
T(-413)A polymorphism and CHD & S versus L & 0.068 \\
& SS versus SL+LL & 0.366 \\
& SS+SL versus LL & 0.127 \\
& SL versus LL & 0.133 \\
& SS versus LL & 0.142 \\
& A versus T & 0.395 \\
& AA versus AT+TT & 0.263 \\
& AA+AT versus TT & 0.820 \\
\hline
\end{tabular}
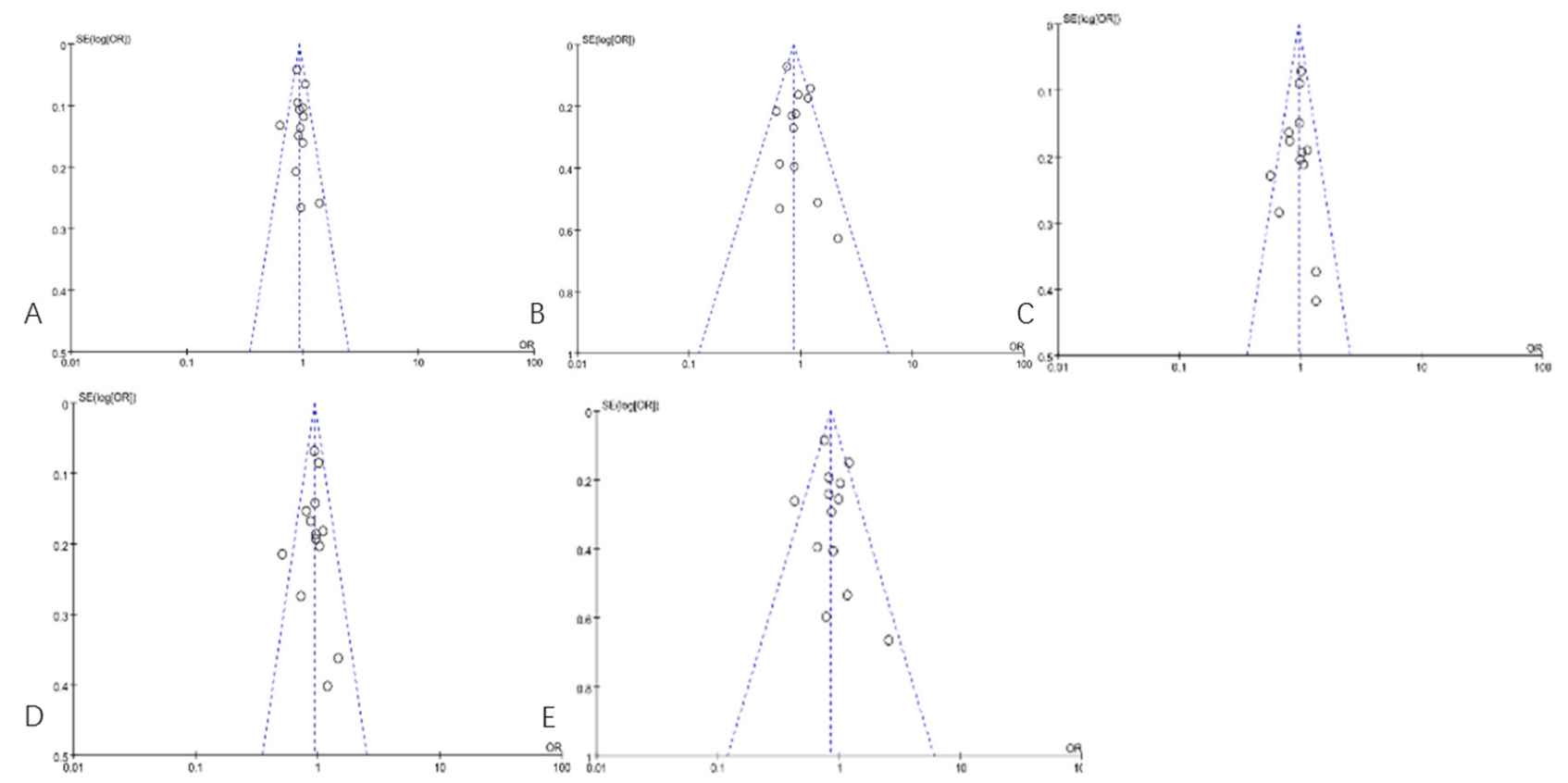

Figure 9: Funnel plot of the association between the (GT)n polymorphism in the HO-1 gene and CHD risk. A. the allele model (S/L); B. the recessive model (SS/SL+LL); C. the dominant model (SS+SL/LL); D. the co-dominant model (SL/LL); E. the codominant model (SS/LL). 


\section{DISCUSSION}

The present study indicates that HO-1 gene polymorphisms are associated with CHD independently. There are three isoforms of heme oxygenase in human, including HO-1, HO-2 and HO-3. HO-1 is up-regulated by oxidative stress and its own substrate heme [43] and may be modulated by fragile histidine triad gene (FHIT) [44]. Animal experiments and clinical trials have confirmed that the HO-1 enzyme is expressed in various tissues and cells, including asatherosclerotic lesions and vascular smooth muscle cells [43]. Therefore, HO-1 is considered to provide protective functions against asatherosclerotic lesions formation [43] and cellular proliferation [45].

Recently, many studies have suggested that HO-1 gene polymorphisms were associated with CHD [9-20]. The SS genotype of the HO-1(GT)n locus may promote HO-1 gene expression and result in increased protein production, thereby raising bilirubin levels and so reducing the risk of CHD [9-16] and restenosis following coronary stenting [22, 23]. However, some studies have come to other conclusions. Theoretically, meta-analysis can clarify the conclusions. Unfortunately, the two previously published meta-analyses came to different conclusions. Recently several new publications focused on this topic have been published. We conducted an update meta-analysis to clarify the association of HO-1 polymorphisms with CHD and RS.

In the present study, a significant association of the (GT)n SS genotype or S allele with decreased the risk of CHD and RS after PCI was observed. In the subgroup analysis, the Asian population showed a positive association in the all genetic models, while the study conducted with the Caucasian population showed no significant association in any of the genetic models. This can be explained by the high prevalence of $\mathrm{S}$ allele in Asian subjects. In addition, because of difference in life styles, ethnicity, region, and other factors, there are large differences in gene distribution. Nevertheless, more studies have been included in the present meta-analysis, and all the included studies were of high quality according to the methodological quality assessment. No significant heterogeneity was identified, and supplementary analysis, including subgroup and sensitivity analysis, were performed to strengthen our conclusions.

The present study also shows an association between genetic factors and the risk of stenting RS. We found that the HO-1T(-413)A SNP was associated with decreased risk for CHD. However, this significant association should be interpreted cautiously. First, CHD or RS after PCI are complex diseases with multifactorial etiology, including gene and environmental factors. Only one SNP is not sufficient to provide the appropriate explanation of genetic risk for CHD or RS after PCI.
Gene-gene or gene-environment interaction factors may influence the risk of a subject for CHD or RS. Second, some potentially confounding factors should be discussed. Primary sources of heterogeneity include the following: the condition of the population included in this study, the main characteristics of the stents following PCI and the treatment compliance of the patients. In addition, the number of included studies for the HO-1T(-413)A SNP is small, and so we did not perform further subgroup analysis in the present study. Even so, the conclusion still give us some information on the pathogenesis of CHD and RS risk factors. Indeed, $\mathrm{HO}-1$ is involved in the occurrence of restenosis by inhibiting vascular smooth muscle cells, attenuating vascular remodeling, and other mechanisms [48, 49]. Although in our meta-analysis, we found that $\mathrm{S}$ allele carriers have decreased risk for RS after PCI compare with $\mathrm{L}$ allele carriers and that the HO-1 T(-413)A SNP was associated with decreased risk of CHD, the importance of HO-1 in human RS following coronary stenting has not been fully defined.

Several limitations of our study need to be considered. First, the number of included studies for HO-1T(-413)A SNP is small, and so we did not perform further subgroup analyses in the present study. Second, limiting the included studies to those published in English and Chinese might have missed some eligible studies in other languages. In addition, it is possible that the results included in the present meta-analysis are affected by miscounting the genotypes or misclassification of CHD and RS.

Hence, our results suggested that carrying the $\mathrm{S}$ allele of the (GT)n locus or the A allele of the T(-413) A locus in the HO-1 gene promoter decreased the risk of CHD. We also found that carrying shorter (GT)n repeats (S or SS genotype) but not the T(-413)A SNP was associated with decreased risk of RS after PCI. These effects appeared more significant in Asian populations.

\section{MATERIALS AND METHODS}

\section{Identification of eligible studies}

We carried out a systematic search in PubMed, Web of Science, the Cochrane Library, Wanfang Data and CNKI (China National Knowledge Infrastructure), with the last search updated on February 1, 2016. The following terms were used: "heme oxygenase 1" or "HO-1" or "HMOX1 " and "polymorphism" and "coronary artery disease" or "cardiac heart disease" or "myocardial infarction" or "MI" or "angina pectoris" or "arteriosclerosis" or "coronary disease" or "acute coronary syndrome" or "coronary stenosis" or "restenosis" or "stent-restenosis". We included literature on relevant studies carried out in human subjects published in English and Chinese. CHD was defined as confirmed myocardial infarction, typical angina pectoris 
(by the World Health Organization criteria), or a history of PCI or as diagnosed by angiography. The controls were defined as in-patients, outpatients, or members of the general population who were without typical angina pectoris or electrocardiographic abnormality and without coronary artery stenosis of more than $20 \%$ upon coronary angiography [28].

\section{Inclusion criteria}

The studies in our meta-analysis met the following inclusion criteria: (1) case-control or cohort studies; (2) investigation of the association between the HO-1 gene polymorphisms and coronary artery disease or coronary restenosis; (3) inclusion of all patients, which were using drug-eluting stents(DES) and had 6 months follow-up angiography, after stenting (Restenosis, was defined as angiographic restenosis, diameter stenosis of $>50 \%$, and clinical restenosis, target vessel revascularization during the follow-up.);(4) studies focusing on humans; and (5) unabridged genotype data could be acquired to calculate the odds ratios (ORs) and 95\% confidence intervals (CIs).

\section{Exclusion criteria}

We excluded papers according to the following criteria: (1) studies with no genotype data; (2) commentaries, reviews and editorials; (3) family-based studies of pedigrees; and (4) repeated studies using the same population data or duplicated data.

\section{Data extraction}

Data collection from the eligible studies were conducted independently by two investigators (Zhang and Zheng). The following contents were collected: name of the first author, year of publication, ethnicity or geographic location of the study subjects, the characteristics of cases and controls, genotyping methods, number of cases and controls, the criteria for cases and controls, genotype frequency in cases and controls for HO-1 genotypes, Hardy-Weinberg equilibrium, and type of stents. Two investigators checked the extracted data and reached a consensus on all the data. If a disagreement existed, a third investigator (Xie) would adjudicate the disagreement.

\section{Quality assessment}

To determine the methodological quality of each study, we used the Newcastle-Ottawa scale (NOS), which uses a "star" rating system to judge the quality of all included studies. The NOS ranges between zero (worst) and nine stars (best). Studies with a score equal to or higher than seven were considered to be of good quality. A score equal to or higher than four and less than seven was regarded as being poor quality. Two investigators (Zheng and Zhang) independently assessed the quality of included studies, and the results were reviewed by a third investigator (Xie). Disagreement was resolved by discussion.

\section{Statistics analysis}

Stata 12.0 software (StataCorp, College Station, TX, USA) was used for statistical analysis in our metaanalysis. The Hardy-Weinberg equilibrium (HWE) was calculated for each study using the Chi-square test in control groups, and $\mathrm{P}<0.05$ was considered a significant deviation from the HWE. Odds ratios and $95 \%$ confidence intervals were calculated to assess the strength of the associations of the polymorphism and susceptibility to CHD or RS risk. The associations between the genetic variant and $\mathrm{CHD}$ or RS risk of pooled ORs were performed for allelic comparison [(GT) n: S vs. L; T(-413)A: A vs. T], a recessive genetic model [(GT) n: SS vs. SL+LL; T(-413)A: AA vs. AT+TT], a dominant model $[(\mathrm{GT}) \mathrm{n}: \mathrm{SS}+\mathrm{SL}$ vs. LL; T(-413)A: AA+AT vs. TT], and a co-dominant model [(GT) n: SL vs. LL, SS vs. LL; T(-413)A: AT vs. TT, AA vs. TT]. The statistically significant level was determined by Z-test, and significance was set at $p<0.05$. Heterogeneity was assessed using the $\mathrm{H}$ test (significance level of $P<0.1$ ) and the $\mathrm{I}^{2}$ test (greater than $50 \%$ as evidence of significant inconsistency). Pooled effect sizes were determined using a fixed-effects model (the Mantel-Haenszel method) when heterogeneity was negligible $\left(\mathrm{I}^{2}<50 \%\right)$ or a random-effects model (the DerSimonian and Kacker method) when significant heterogeneity was present $\left(\mathrm{I}^{2} \geq 50 \%\right)$. We also performed a sensitivity analysis to evaluate the effect of each study on the combined ORs by omitting each study in turn. Finally, we utilized Egger's tests to assess the potential publication bias.

\section{ACKNOWLEDGMENTS AND FUNDING}

This work was supported by the National Natural Science Foundation of China (81560070).

\section{CONFLICTS OF INTEREST}

We state that we have no conflicts to disclose.

\section{REFERENCES}

1. Sabatine MS1, Seidman JG, Seidman CE. Cardiovascular genomics.Circulation. 2006; 113:e450-455.

2. Kastrati A, Mehilli J, von Beckerath N, Dibra A, Hausleiter J, Pache J, Schühlen H, Schmitt C, Dirschinger J, Schömig A; ISAR-DESIRE Study Investigators.Sirolimus-eluting stent or paclitaxel-eluting stent vs balloon angioplasty for 
prevention of recurrences in patients with coronary in-stent restenosis: a randomized controlled trial.JAMA. 2005; 293:165-71

3. Kastrati A, Koch W, Berger PB, Mehilli J, Stephenson K, Neumann FJ, von Beckerath N, Böttiger C, Duff GW, Schömig A. Protective role against restenosis from an interleukin-1 receptor antagonist gene polymorphism in patients treated with coronary stenting. J Am Coll Cardiol. 2000; 36:2168-73.

4. Pendyala L, Jabara R, Shinke T, Chronos N, Robinson $\mathrm{K}$, Li J, Hou D.Drug-eluting stents: present and future. Cardiovasc Hematol Agents Med Chem. 2008; 6:105-15.

5. Baranano DE, Rao M, Ferris CD, Snyder SH. Biliverdin reductase: a major physiologic cytoprotectant. Proc Natl Acad Sci U S A. 2002; 99:16093-8.

6. Lüblinghoff N1, Winkler K, Winkelmann BR, Seelhorst U, Wellnitz B, Boehm BO, März W, Hoffmann MM. Genetic variants of the promoter of the heme oxygenase-1 gene and their influence on cardiovascular disease (the Ludwigshafen Risk and Cardiovascular Health study). BMC Med Genet. 2009; 10:36.

7. Dick P, Schillinger M, Minar E, Mlekusch W, Amighi J, Sabeti S, Schlager O, Raith M, Endler G, Mannhalter C, Wagner O, Exner M. Haem oxygenase-1 genotype and cardiovascular adverse events in patients with peripheral artery disease. Eur J Clin Invest. 2005; 35:731-737.

8. Brydun A, Watari Y, Yamamoto Y, Okuhara K, Teragawa H, Kono F, Chayama K, Oshima T, Ozono R. Hypertens Res. Reduced expression of heme oxygenase- 1 in patients with coronary atherosclerosis. 2007; 30:341-348.

9. Chen YH, Kuo KL, Hung SC, Hsu CC, Chen YH, Tarng DC. Length polymorphism in heme oxygenase-1 and risk of CKD among patients with coronary artery disease. J Am Soc Nephrol. 2014; 25:2669-2677.

10. Chen M, Zhou L, Ding H, Huang S, He M, Zhang X, Cheng L, Wang D, Hu FB, Wu T. Short (GT) (n) repeats in heme oxygenase- 1 gene promoter are associated with lower risk of coronary heart disease in subjects with high levels of oxidative stress. Cell Stress Chaperones. 2012; 17:329-38.

11. Zhang GH, Mai RQ, Chen SM, et al. Association of (GT) $\mathrm{n}$ polymorphism in heme oxygenase-1 gene promoter and susceptibilityof coronary heart disease in Chaoshan population [in Chinese]. Chin J Arterioscler, 2010; 18:807-809.

12. Wang YH, Ma YT, Fu ZY, et al. Association between genetic polymorphism in the promoter region of heme oxygenase-1 and myocardial infarction in patients from Uighur ethnics of Xinjiang Autonomous Region [inChinese]. Chin J Emerg Med, 2009; 18:719-722.

13. Chen YH, Chau LY, Chen JW, Lin SJ. Serum bilirubin and ferritin levels link heme oxygenase-1 gene promoter polymorphism and susceptibility to coronary artery disease in diabetic patients. Diabetes Care. 2008; 31:1615-1620.

14. Funk M, Endler G, Schillinger M, Mustafa S, Hsieh K, Exner M, Lalouschek W, Mannhalter C, Wagner O. The effect of a promoter polymorphism in the heme oxygenase-1 gene on the risk of ischaemic cerebrovascular events: the influence of other vascular risk factors. Thromb Res. 2004; 113:217-223.

15. Kaneda H, Ohno M, Taguchi J, Togo M, Hashimoto H, Ogasawara K, Aizawa T, Ishizaka N, Nagai R. Heme oxygenase-1 gene promoter polymorphism is associated with coronary artery disease in Japanese patients with coronary risk factors. Arterioscler Thromb Vasc Biol. 2002; 22:1680-1685.

16. Schillinger M, Exner M, Mlekusch W, Domanovits H, Huber K, Mannhalter C, Wagner O, Minar E. Heme oxygenase-1 gene promoter polymorphism is associated with abdominal aortic aneurysm. Thromb Res. 2002; 106:131-136.

17. Han SW, Song W, Kim HS, Shin KS, Kang H, Cho HC, Ki CS, Park MJ. HMOX1 gene promoter polymorphism is not associated with coronary artery disease in Koreans. Ann Lab Med. 2014; 34:337-44.

18. Gregorek AC1, Gornik KC, Polancec DS, Dabelic S. GT microsatellite repeats in the heme oxygenase-1 gene promoter associated with abdominal aortic aneurysm in Croatian patients. Biochem Genet. 2013; 51:482-492.

19. Lüblinghoff N, Winkler K, Winkelmann BR, Seelhorst U, Wellnitz B, Boehm BO, März W, Hoffmann MM. Genetic variants of the promoter of the heme oxygenase-1 gene and their influence on cardiovascular disease (the Ludwigshafen Risk and Cardiovascular Health study). BMC Med Genet. 2009; 10:36.

20. Endler G, Exner M, Schillinger M, Marculescu R, SunderPlassmann R, Raith M, Jordanova N, Wojta J, Mannhalter C, Wagner OF, Huber K. A microsatellite polymorphism in the heme oxygenase-1 gene promoter is associated with increased bilirubin and HDL levels but not with coronary artery disease. Thromb Haemost. 2004; 91:155-161.

21. Ono K, Goto Y, Takagi S, Baba S, Tago N, Nonogi H, Iwai $\mathrm{N}$. A promoter variant of the heme oxygenase-1 gene may reduce the incidence of ischemic heart disease in Japanese. Atherosclerosis. 2004; 173:315-319.

22. Schillinger M, Exner M, Minar E, Mlekusch W, Müllner M, Mannhalter C, Bach FH, Wagner O. Heme oxygenase-1 genotype and restenosis after balloon angioplasty: a novel vascular protective factor. J Am Coll Cardiol. 2004; 43:950-957.

23. Chen YH, Chau LY, Lin MW, Chen LC, Yo MH, Chen JW, Lin SJ. Heme oxygenase-1 gene promotor microsatellite polymorphism is associated with angiographic restenosis after coronary stenting. Eur Heart J. 2004; 25:39-47.

24. Tiroch K, Koch W, von Beckerath N, Kastrati A, Schömig A. Heme oxygenase-1 gene promoter polymorphism and 
restenosis following coronary stenting. Eur Heart J. 2007; 28:968-73.

25. Wijpkema JS, van Haelst PL, Monraats PS, Bruinenberg M, Zwinderman AH, Zijlstra F, van der Steege G, de Winter RJ, Doevendans PA, Waltenberger J, Jukema JW, Tio RARestenosis after percutaneous coronary intervention is associated with the angiotensin-II type-1 receptor $1166 \mathrm{~A} / \mathrm{C}$ polymorphism but not with polymorphisms of angiotensin-converting enzyme, angiotensin-II receptor, angiotensinogen or heme oxygenase-1.. Pharmacogenet Genomics. 2006; 16:331-7.

26. Exner M, Schillinger M, Minar E, Mlekusch W, Schlerka G, Haumer M, Mannhalter C, Wagner O. Heme oxygenase-1 gene promoter microsatellite polymorphism is associated with restenosis after percutaneous transluminal angioplasty. J Endovasc Ther. 2001; 8:433-440.

27. Qiao H, Sai X, Gai L, Huang G, Chen X, Tu X, Ding Z. Association between heme oxygenase 1 gene promoter polymorphisms and susceptibility to coronary artery disease: a HuGE review and meta-analysis. Am J Epidemiol. 2014; 179:1039-1048.

28. Rong Y, Bao W, Shan ZL, Tiroch K, Koch W, von Beckerath N, Kastrati A, Schömig A. Heme oxygenase-1 gene promoter polymorphisms and risk of coronary artery disease and coronary restenosis: A meta-analysis [abstract]. Eur Heart J Suppl, 2012; 14:P039.

29. Song F, Li X, Zhang M, Yao P, Yang N, Sun X, Hu FB, Liu L. Association between heme oxygenase-1 gene promoter polymorphisms and type 2 diabetes in a Chinese population. Am J Epidemiol. 2009; 170:747-56.

30. Dick P, Schillinger M, Minar E, Mlekusch W, Amighi J, Sabeti S, Schlager O, Raith M, Endler G, Mannhalter C, Wagner O, Exner M. Haem oxygenase-1 genotype and cardiovascular adverse events in patients with peripheral artery disease. Eur J Clin Invest. 2005; 35:731-737.

31. Arredondo M, Jorquera D, Carrasco E, Albala C, Hertrampf E. Microsatellite polymorphism in the heme oxygenase-1 gene promoter is associated with iron status in persons with type 2 diabetes mellitus. Am J Clin Nutr. 2007; 86:1347-1353.

32. Wu MM, Chiou HY, Chen CL, Wang YH, Hsieh YC, Lien LM, Lee TC, Chen CJ.GT-repeat polymorphism in the heme oxygenase-1 gene promoter is associated with cardiovascular mortality risk in an arsenic-exposed population in northeastern Taiwan. Toxicol Appl Pharmacol. 2010; 248:226-233.

33. Liang KW, Sheu WH, Lee WL, Lee IT, Lin SY, Ting CT, Lee WJ. Shorter GT repeats in the heme oxygenase-1 gene promoter are associated with a lower severity score in coronary artery disease. J Chin Med Assoc. 2013; 76:312-8.

34. Holweg CT, Balk AH, Uitterlinden AG, Niesters HG, Maat LP, Weimar W, Baan CC. Functional heme oxygenase-1 promoter polymorphism in relation to heart failure and cardiac transplantation. J Heart Lung Transplant. 2005; 24:493-497.

35. Dick P, Schillinger M, Minar E, Mlekusch W, Amighi J, Sabeti S, Schlager O, Raith M, Endler G, Mannhalter C, Wagner O, Exner M. Haem oxygenase-1 genotype and cardiovascular adverse events in patients with peripheral artery disease. Eur J Clin Invest. 2005; 35:731-737.

36. Chen YH, Lin SJ, Lin MW, Tsai HL, Kuo SS, Chen JW, Charng MJ, Wu TC, Chen LC, Ding YA, Pan WH, Jou YS, Chau LY. Microsatellite polymorphism in promoter of heme oxygenase-1 gene is associated with susceptibility to coronary artery disease in type 2 diabetic patients. Hum Genet. 2002; 11:1-8.

37. Lin R, Wang Y, Wang Y, Fu W, Zhang D, Zheng H, Yu T, Wang Y, Shen M, Lei R, Wu H, Sun A, Zhang R, Wang X, Xiong M, Huang W, Jin L. Common variants of four bilirubin metabolism genes and their association with serum bilirubin and coronary artery disease in Chinese Han population. Pharmacogenet Genomics. 2009; 19:310-318.

38. Bai CH, Chen JR, Chiu HC, Chou CC, Chau LY, Pan WH. Shorter GT repeat polymorphism in the heme oxygenase-1 gene promoter has protective effect on ischemic stroke in dyslipidemia patients. J Biomed Sci. 2010; 17:12.

39. Bao W, Song F, Li X, Rong S, Yang W, Wang D, Xu J, Fu J, Zhao Y, Liu L. Association between heme oxygenase-1 gene promoter polymorphisms and type 2 diabetes mellitus: a HuGE review and meta-analysis. Am J Epidemiol. 2010; 172:631-636.

40. Pechlaner R, Willeit P, Summerer M, Santer P, Egger G, Kronenberg F, Demetz E, Weiss G, Tsimikas S, Witztum JL, Willeit K, Iglseder B, Paulweber B, Kedenko L, Haun M, Meisinger C, Gieger C, Müller-Nurasyid M, Peters A, Willeit J, Kiechl S. Heme oxygenase-1 gene promoter microsatellite polymorphism is associated with progressive atherosclerosis and incident cardiovascular disease. Arterioscler Thromb Vasc Biol. 2015; 35:229-236.

41. Wu MM, Chiou HY, Chen CL, Hsu LI, Lien LM, Wang $\mathrm{CH}$, Hsieh YC, Wang YH, Hsueh YM, Lee TC, Cheng WF, Chen CJ. Association of heme oxygenase-1 GT-repeat polymorphism with blood pressure phenotypes and its relevance to future cardiovascular mortality risk: an observation based on arsenic-exposed individuals. Atherosclerosis. 2011; 219:704-708.

42. Wu MM, Chiou HY, Chen CL, Wang YH, Hsieh YC, Lien LM, Lee TC, Chen CJ.GT-repeat polymorphism in the heme oxygenase-1 gene promoter is associated with cardiovascular mortality risk in an arsenic-exposed population in northeastern Taiwan. Toxicol Appl Pharmacol. 2010; 248:226-233.

43. Bouche D, Chauveau C, Roussel JC, Mathieu P, Braudeau C, Tesson L, Soulillou JP, Iyer S, Buelow R, Anegon I. Inhibition of graft arteriosclerosis development in rat aortas following heme oxygenase-1 gene transfer. Transpl Immunol. 2002; 9:235-238. 
44. Boylston JA, Brenner C. A knockdown with smoke model reveals FHIT as a repressor of Heme oxygenase 1. Cell Cycle. 2014; 13:2913-30.

45. Juan SH, Lee TS, Tseng KW, Liou JY, Shyue SK, Wu KK, Chau LY.Adenovirus-mediated heme oxygenase-1 gene transfer inhibits the development of atherosclerosis in apolipoprotein E-deficient mice.Circulation. 2001; 104:1519-1525.

46. Morita T. Heme oxygenase and atherosclerosis. Arterioscler Thromb Vasc Biol. 2005; 25:1786-1795.

47. Taha H, Skrzypek K, Guevara I, Nigisch A, Mustafa S, Grochot-Przeczek A, Ferdek P, Was H, Kotlinowski J, Kozakowska M, Balcerczyk A, Muchova L, Vitek L,
Weigel G, Dulak J, Jozkowicz A. Role of heme oxygenase-1 in human endothelial cells: lesson from the promoter allelic variants. Arterioscler Thromb Vasc Biol. 2010; 30:1634-1641.

48. Okuhara K, Kisaka T, Ozono R, Kurisu S, Inoue I, Soga J, Yano Y, Oshima T, Kihara Y, Yoshizumi M. Change in bilirubin level following acute myocardial infarction is an index for heme oxygenase activation. South Med J. 2010; 103:876-881.

49. Peyton KJ, Reyna SV, Chapman GB, Ensenat D, Liu XM, Wang H, Schafer AI, Durante W. Heme oxygenase1-derived carbon monoxide is an autocrine inhibitor of vascular smooth muscle cell growth.Blood. 2002; 99:4443-4448. 